\section{A general purpose analysis of variance routine}

JAMES R. ULLRICH

University of Montana, Missoula, Montaria 59801

and

GORDON F. PITZ

Southern Illinois University, Carbondale, Illinois 62901

This program performs a wide variety of completely randomized factorial, randomized block factorial, and split-plot factorial analyses of variance. It accepts data from any of these designs which have up to eight factors, four of which may be independent (between blocks or between subjects) and four of which may be repeated (within blocks or within subjects). The sample size in each group is not required to be equal as long as it is proportional across subgroups. In addition to performing the indicated analysis of variance, the routine performs an $F_{\max }$ test for the heterogeneity of variance and Chi-square tests for the equality and symmetry of the covariance matrices.

The form of the parameter cards necessary for this program and the manner in which the data must be input are easy to learn and use. Extensive diagnostics are incorporated into the program as an aid to users who may not have provided the correct parameters or may have attempted an illegitimate design. The program has been in general use at a number of universities and appears to be very reliable and error free.

This program complements existing routines such as Butler, Kamlet, and Monty (1969) and the BMD series (Dixon, 1973) by allowing some unequal $\mathrm{N}$ designs, by optionally performing one of a number of transformations of the data, and by performing checks on the assumptions of homogeneity of variance and equality and symmetry of the covariance matrices. Probability levels for the $F$ ratios are computed and there is a convenient option for the labeling of the factors.

Method. A pseudofactor corresponding to blocks is introduced and treated as an additional ninth factor. The additional factor is considered random; all others are considered fixed. The program performs a splitplot factorial analysis with the assumption that there is an $\mathrm{N}$ of 1 in each cell. Thus, for a two-factor design with two levels of an independent factor (between blocks or between subjects) and three levels of a repeated factor (within blocks or within subjects) with 10 subjects in each of the two groups, the program internally performs a 2 by 10 by 3 split-plot factorial analysis of variance with an $\mathrm{N}$ of 1 . In this three-way design, the factors with 2 and 10 levels are considered independent and the factor with 3 levels is considered repeated. The blocks factor with 10 levels is random; the others are fixed. The output provided by the program provides values of the means, sums of squares, mean squares, $F$ ratios, and probability levels appropriate to the original 2 by 3 design.

Limitations. Any number of levels for any of the factors and any number of blocks can be accepted subject to the limitations of available memory. Currently, the program has a maximum of 5,000 observations and a maximum size for the variancecovariance matrix of 25 by 25 . These limitations may be easily changed by minor modifications of the array sizes within the program.

Source language and computer. The 700-line program is written in a neutral dialect of FORTRAN IV and has been run on a DEC System 10, an IBM 360/370 and a Xerox Sigma 7. The program is being translated into BASIC and this translation should be available shortly.

Availability. The source code, an extensive users' manual, test data, and output appropriate to a number of designs in Kirk (1968) and Winer (1971) may be obtained from the first author at no cost.

\section{REFERENCES}

Butler, D. H., Kamlet, A. S., \& Monty, P. A. A Multipurpose Analysis of Variance Computer Program. Psychonomic Monograph Supplements. Vol. 2. No. 16. (whole No. 32), 1969.

Dixon, W. J. (Ed.) BMD Biomedical Computer Programs. Berkeley: University of California Press, 1973.

KIRK, R. E. Experimental design: Procedures for the behavioral sciences. Belmont, Calif: Brooks/Cole, 1968.

WINER, B. J. Statistical principles in experimental design (2nd ed.). New York: McGraw-Hill, 1971. 